\title{
Dynamics of nonlinear coupled electrostatic micromechanical resonators under two-frequency parametric and external excitations
}

\author{
Wen-Ming Zhang*, Guang Meng and Ke-Xiang Wei \\ State Key Laboratory of Mechanical System and Vibration, School of Mechanical Engineering, Shanghai Jiao Tong \\ University, 800 Dongchuan Road, Shanghai 200240, P.R. China
}

Received 8 May 2009

Revised 31 August 2009

\begin{abstract}
In this paper, nonlinear dynamics and chaos of electrostatically actuated MEMS resonators under two-frequency parametric and external excitations are investigated analytically and numerically. A nonlinear mass-spring-damping model is used to accounting for squeeze film damping and the parallel plate electrostatic force. The micro-structure is excited by a dc bias electrostatic force and a harmonic force with a frequency tuned closely to their fundamental natural frequencies (combination oscillation). The quality factor is calculated for the microcantilever beam of the resonator considering squeeze film damping. The effect of nonlinear squeeze film damping on the frequency response, quality factor, resonant frequency and nonlinear dynamic characteristics of the dynamic system are provided with numerical simulations using the bifurcation diagram, Poicare maps, largest Lyapunov exponent and phase portrait. The results show that the dynamic system goes through a complex nonlinear vibration as the system parameters change. It is indicated that the effect of nonlinear squeeze film damping should be considered due to its decreasing the quality factor and changing the nonlinear phenomena of the MEMS resonators.
\end{abstract}

Keywords: MEMS, resonator, electrostatic actuation, squeeze film damping, combination oscillation

\section{Introduction}

Recent technological advances have enabled the fabrication of resonators down to micro- and even nano- scales. The relevance of nanotechnology is well recognized, so new developments and applications based on nonlinear dynamics, chaos, fractals, and nonlinear control, are nowadays reached in an interdisciplinary framework [1,2]. Resonant MEMS devices are widely used to measure strain, force or displacement [3-5]. Electrostatically actuated resonators have the advantages of simple structures that allow easy batch fabrications and they form a major component in many MEMS devices, such as capacitive switch [3], filter [4], and mass sensor [5].

During the past decade, many researchers had paid attention to the applications of parametric excitation for MEMS/NEMS devices [6-26]. Carr et al. [6] and Zalalutdinov et al. [7] studied the parametric amplification of the motion of resonators through electrostatic and optical actuation. However, the reported methods should be used to discuss the instability and control strategies. It is necessary to benefit from the parametric amplification phenomena which had not remarked. A demonstration of the phenomena of parametrically excited vibrations in MEMS/NEMS

*Corresponding author. Tel.: +86 213420 6813-818; E-mail: wenmingz@ sjtu.edu.cn. 
was reported in [8,17]. Abdel-Rahman and Nayfeh [9] and Gallacher et al. [10] applied the super-harmonic and combination parametric resonances as suitable excitation methods to minimize electrical "feedthrough". The phenomenon of the parametric excitation in spatially extended MEMS/NEMS was investigated theoretically by Lifshitz and Cross [11]. Gallacher et al. [12] studied the application of combined external forcing and parametric excitation to the micro-ring gyroscope in order to enable parametric amplification of the dynamic gain of the primary mode by at least two orders of magnitude. Napoli [13] gave the dynamic responses of a parametrically excited micro-cantilever using the linear and nonlinear Mathieu equations. The study on the array of parametrically excited strings were carried out both experimentally [14] and theoretically [11]. Hu et al. [15] discussed the resonances of electrostatically actuated micro-cantilevers, while Baskaran and Turner [16] demonstrated the coupled modes parametric resonance. It should be noted, however, that an important feature of parametric excitation is the ability of stabilizing a statically unstable system. Krylov et al. [18] investigated the possibility of parametric stabilization of electrostatically actuated microstructures under the effects of ac component and dc component voltages. Younis and Nayfeh [19] and Abdel-Rahman and Nayfeh [9] studied the dynamic responses of an electrostatically resonator to a primary-resonance excitation [19], a superharmonic-resonance excitation of order two [9], and a subharmonicresonance excitation of order one-half [9]. It can be concluded that these models gave accurate results for small ac amplitudes and hence small motions. Younis et al. [3] applied the shooting method to simulate the dynamics of a novel MEMS switch excited near primary resonance and undergoing large motion. Mestrom et al. [23] modelled the dynamics of a MEMS resonator numerically and experimentally considering the effect of thermal noise. Kacem et al. [24] studied the nonlinear dynamics of nanomechanical beam resonators to improve the performance of NEMS-based sensors. Alsaleem et al. [25] investigated the nonlinear phenomena, including primary resonance, superharmonic and subharmonic resonances, in electrostatically actuated resonators both experimentally and theoretically. However, the dynamic response of the electrostatic MEMS resonator to a combination resonance excitation has not been paid more attention in literature. This work aims at extending our previous studies $[20,22$, 26] and is a step toward modeling and understanding of the nonlinear dynamics of the electrostatically actuated resonator under combination resonant condition with the effect of nonlinear squeeze film damping.

The outline for this paper is as follows. In Section 2, the simplified mass-spring-damping dynamic model of an electrostatically actuated MEMS resonator is presented. In Section 3, perturbation analyses of the governing equation using multiple scale method is given. Additionally, numerical simulations and results of the resonant frequencies, frequency responses, quality factors and nonlinear dynamic properties are described and discussed in Section 4. Finally, some conclusions are drawn in Section 5.

\section{Dynamic model}

De and Aluru [27,28] suggested that it is easier to understand the physics behind the dynamic properties of electrostatic MEMS with a simpler mass-spring-damping model. Therefore, a microcantilever beam was considered in this work and the electrostatically actuated MEMS structure was $4.5 \mathrm{um} \times 80 \mathrm{um} \times 200 \mathrm{um}$ in dimensions [29], and the dynamic model was shown in Fig. 1. The simplified mass-spring-damping system can be written as

$$
m \ddot{x}(t)+c \dot{x}(t)+k x(t)+k_{c} x(t)^{3}=F_{e}(x, t)+F_{s}(x, \dot{x})
$$

where $m$ is the mass, $k$ and $c$ are the effective spring stiffness and damping coefficient of the simplified system, respectively, $k_{c}$ is the cubic nonlinear stiffness, and $F_{s}$ and $F_{e}$ are the squeeze film damping force and nonlinear electrostatic force, respectively. The electrostatic force $F_{e}$ is given by

$$
F_{e}(x, t)=\frac{\varepsilon_{0} A_{0}}{2}\left(\frac{1}{d_{0}^{2}}+\frac{2}{d_{0}^{3}} x\right) V^{2}(t)
$$

where the input voltage $V(t)=V_{d c}+V_{a c} \cos \omega t$, in which $V_{d c}$ and $V_{a c}$ are dc bias voltage and alternating current (ac) voltage, respectively, $\varepsilon_{0}$ is the absolute dielectric constant of vacuum $\left(\varepsilon_{0}=8.85 \times 10^{-12} \mathrm{~F} / \mathrm{m}\right), A_{0}$ is the overlapping area between the two plates, and $d_{0}$ is the gap.

The surrounding gas medium plays an important role on the dynamics of the cantilever in MEMS resonators. For a rectangular cantilever beam [30], when the quality factor of the fundamental mode of the cantilever in gas exceeds 


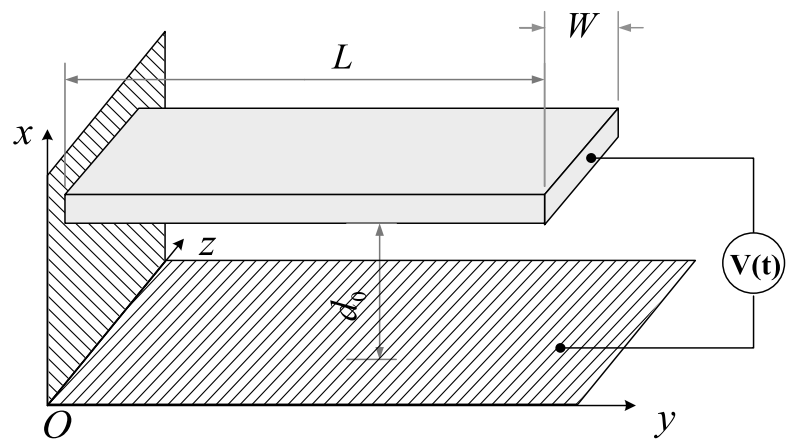

(a) microcantilever beam resonator schematic dc bias voltage $V_{d c}$

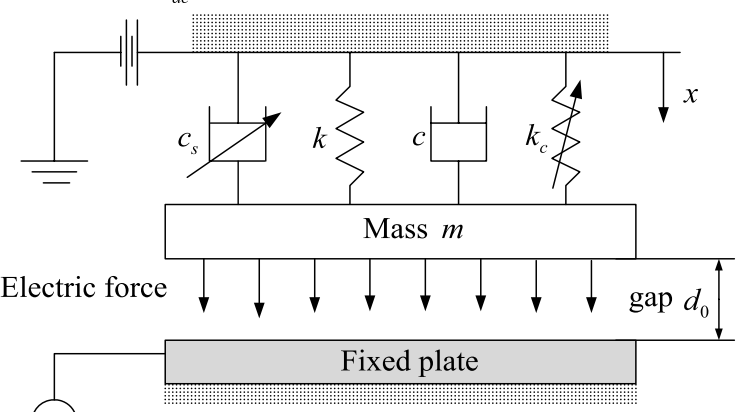

ac voltage

$V_{a c} \cos (\omega t)$

(b) dynamic model schematic

Fig. 1. Simplified dynamic model of a micromechanical electrostatic resonator.

1, which is typically satisfied when the cantilever is placed in air, the relationship between the vacuum resonant frequency $\omega_{v a c}$ and the resonant frequency in gas $\omega_{\text {gas }}$ can be given by [31]

$$
\frac{\omega_{\text {gas }}}{\omega_{\text {vac }}}=\left(1+\frac{\pi \rho B}{4 \rho_{c} H} \Gamma_{r}\left(\omega_{\text {gas }}\right)\right)^{-1 / 2}=\left(1+\frac{\pi}{4} \Pi \Gamma_{r}\left(\omega_{\text {gas }}\right)\right)^{-1 / 2}
$$

where $\rho$ is the density of the gas, the nominal width $B$ is the dominant length scale in the gas flow, $\rho_{c}$ and $H$ are the density and thickness of the cantilever, respectively, and the natural scaling parameter is $\Pi=\frac{\rho B}{\rho_{c} H}$. The hydrodynamic functions $\Gamma(\omega)$ is defined as $\Gamma=\Gamma_{r}+j \Gamma_{i}[30,31]$, in which $\Gamma_{r}=a_{1}+\frac{a_{2}}{\sqrt{R_{e}}}$ and $\Gamma_{i}=\frac{b_{1}}{\sqrt{R_{e}}}+\frac{b_{2}}{R_{e}}$, and $\mathrm{a}_{1}=1.0553, \mathrm{a}_{2}=3.7997, \mathrm{~b}_{1}=3.8018$, and $\mathrm{b}_{2}=2.7364$ [31].

The quality factor for the bending modes can be written as [31]

$$
Q_{\text {gas }}=\frac{\frac{4 \mu}{\pi \rho B^{2}}+\Gamma_{r}\left(\omega_{\text {gas }}\right)}{\Gamma_{i}\left(\omega_{\text {gas }}\right)}=\frac{\frac{4}{\pi} \cdot \frac{1}{\Pi}+\Gamma_{r}\left(\omega_{\text {gas }}\right)}{\Gamma_{i}\left(\omega_{\text {gas }}\right)}
$$

At a few microns in size, squeeze film damping should be used to represent the gas-film damping experienced by the moving plates $[32,33]$. For a rectangular plate, the squeeze film damping force $F_{s}(x, \dot{x})$ can be given by Taylor series, i.e.

$$
F_{s}(x, \dot{x})=-c_{s}\left(\frac{1}{d_{0}^{3}}+\frac{3}{d_{0}^{4}} x\right) \dot{x}
$$

where the effective damping coefficient $c_{s}=16 \mu C_{r} W^{3} L$, in which $\mu$ is the viscous coefficient, and $W$ and $L$ are the width and length of the cantilever, respectively, and $C_{r}=1-0.6 \frac{W}{L},\left(0<\frac{W}{L}<1\right)$.

The dimensionless variables are defined as

$$
\begin{aligned}
& \tau=\omega_{n} t, \quad \omega_{n}=\sqrt{k / m}, \quad \Omega=\omega / \omega_{n}, \quad \zeta=\frac{c}{m \omega_{n}}, \quad \beta=\frac{c_{s} \omega_{n}}{d_{0}^{2}}, \quad \gamma=\frac{k_{c}}{k} d_{0}^{2}, \quad X=x / d_{0}, \quad \dot{X}=d X / d \tau, \\
& \ddot{X}=d^{2} X / d \tau^{2}, \quad a_{1}=\frac{\varepsilon_{0} A_{0}}{2 m \omega_{n}^{2} d_{0}^{3}}\left(V_{d c}^{2}+\frac{V_{a c}^{2}}{2}\right), \quad a_{2}=\frac{\varepsilon_{0} A_{0}}{m \omega_{n}^{2} d_{0}^{3}} V_{d c} V_{a c}, \quad a_{3}=\frac{\varepsilon_{0} A_{0}}{4 m \omega_{n}^{2} d_{0}^{3}} V_{a c}^{2}
\end{aligned}
$$

Then, the equation of motion can be rewritten in the dimensionless form as

$$
\ddot{X}+\zeta \dot{X}+F_{s}(X, \dot{X})+\left(1-2 a_{1}-2 a_{2} \cos \Omega \tau-2 a_{3} \cos 2 \Omega \tau\right) X+\gamma X^{3}=\sum_{m=1}^{3} a_{m} \cos [(m-1) \Omega \tau]
$$




\section{Perturbation analysis}

Let us consider the weakly nonlinear dynamic system and assume $\zeta=\varepsilon \tilde{\zeta}, \beta=\varepsilon \tilde{\beta}, \gamma=\varepsilon \tilde{\gamma}$ and $a_{m}=\varepsilon \tilde{a}_{m}$ ( $m=1 \sim 3$ ), in which $\varepsilon$ is a small parameter. The equation of the motion has the form

$$
\ddot{X}+X=\varepsilon\left\{\tilde{F}_{s}(X, \dot{X})-\tilde{\gamma} X^{3}-\tilde{\zeta} \dot{X}+2 \sum_{m=1}^{3} \tilde{a}_{m} \cos [(m-1) \Omega \tau] \cdot X+\sum_{m=1}^{3} \tilde{a}_{m} \cos [(m-1) \Omega \tau]\right\}
$$

The desired function $X(\tau)$ can be represented in terms of a series using multiple scale method [34]

$$
X(\tau ; \varepsilon)=X_{0}\left(T_{0}, T_{1}\right)+\varepsilon X_{1}\left(T_{0}, T_{1}\right)+\cdots
$$

where $T_{n}=\varepsilon^{n} \tau$.

Then the first and second derivatives are defined respectively, as follows

$$
\frac{d}{d \tau}=D_{0}+\varepsilon D_{1}+\cdots, \quad \frac{d^{2}}{d \tau^{2}}=D_{0}^{2}+2 \varepsilon D_{0} D_{1}+\cdots
$$

where $D_{n}=\partial /\left(\partial T_{n}\right)$. Substituting Eqs (9) and (8) into (7), equating the coefficient at equal powers of $\varepsilon$, and limiting ourselves by terms of $\varepsilon^{2}$, a set of recurrent equations yield

$$
\begin{aligned}
& D_{0}^{2} X_{0}+X_{0}=\sum_{m=1}^{3} a_{m} \cos \left[(m-1) \Omega T_{0}\right] \\
& D_{0}^{2} X_{1}+X_{1}=-2 D_{0} D_{1} X_{0}-\tilde{\zeta} D_{0} X_{0}+\tilde{F}_{s 0}-\tilde{\gamma} X_{0}^{3}+2 \sum_{m=1}^{3} a_{m} \cos \left[(m-1) \Omega T_{0}\right] \cdot X_{0}
\end{aligned}
$$

where $\tilde{F}_{s 0}$ is the damping term and $\tilde{F}_{s 0}=-\tilde{\beta}\left(D_{0} X_{0}+3 X_{0} D_{0} X_{0}\right)$.

The general solution of Eq. (10) has the form

$$
X_{0}=A \cos \left(T_{0}+\phi\right)+\sum_{k=0}^{2} \Lambda_{k} \cos \left(\omega_{k} T_{0}\right)
$$

where $A=A\left(T_{1}\right)$ and $\phi=\phi\left(T_{1}\right)$ are vibration amplitude and phase, respectively, and $\Lambda_{k}=\frac{a_{k+1}}{1-\omega_{k}^{2}}$ and $\omega_{k}=$ $k \Omega \quad(k=0,1,2)$.

Substituting Eq. (12) into Eq. (11) and eliminating the secular terms from $X_{1}$, for the combination resonant condition, $\omega_{1}+\omega_{2} \approx 1+\varepsilon \sigma$, i.e. $\omega_{2}=2 \omega_{1} \approx \frac{2}{3}$, yields

$$
\left\{\begin{array}{l}
A^{\prime}=-\frac{1}{2} \Gamma_{0} A+\frac{1}{2} \Gamma_{1} \sin \psi \\
A \phi^{\prime}=-\frac{1}{2}\left[2 A a_{1}-\tilde{\gamma} A\left(\frac{3}{4} A^{2}+\Gamma_{2}\right)\right]-\frac{1}{2} \Gamma_{1} \cos \psi
\end{array}\right.
$$

where $\Gamma_{0}=\tilde{\zeta}+\tilde{\beta}+3 \tilde{\beta} \Lambda_{0}, \Gamma_{1}=a_{2} \Lambda_{2}+a_{3} \Lambda_{1}-3 \tilde{\gamma} \Lambda_{0} \Lambda_{1} \Lambda_{2}, \Gamma_{2}=3 \Lambda_{0}^{2}+\frac{3}{2} \Lambda_{1}^{2}+\frac{3}{2} \Lambda_{2}^{2}$ and $\psi=\sigma T_{1}-\phi$.

For the steady-state motion at $a^{\prime}=\psi_{=}^{\prime} 0$, that is the singular points of Eq. (13), the frequency response of the system under combination oscillations can be obtained as follows

$$
\left[4 \Gamma_{0}^{2}+\left(4 \sigma+2 a_{1}-2 \tilde{\gamma} \Gamma_{2}-\frac{3}{2} \tilde{\gamma} A^{2}\right)^{2}\right] A^{2}=4 \Gamma_{1}^{2}
$$

Equation (14) is an implicit equation for the amplitude of the response $A$ as a function of the detuning parameter $\sigma$ and other parameters, including $\gamma, \beta, V_{d c}, V_{a c}$ and $d_{0}$.

\section{Numerical simulation results and discussion}

In this section, numerical simulations on the important dynamic characteristics of the electrostatically actuated MEMS resonator, including resonant frequency, frequency response and nonlinear dynamics, are given in detail. The selected parameters are as follows: $m=3.5 \times 10^{-11} \mathrm{~kg}, k=0.17 \mathrm{~N} / \mathrm{m}, c=1.78 \times 10^{-6} \mathrm{~kg} / \mathrm{s}$ and $A_{0}=$ $1.6 \times 10^{-9} m^{2}[29]$. 


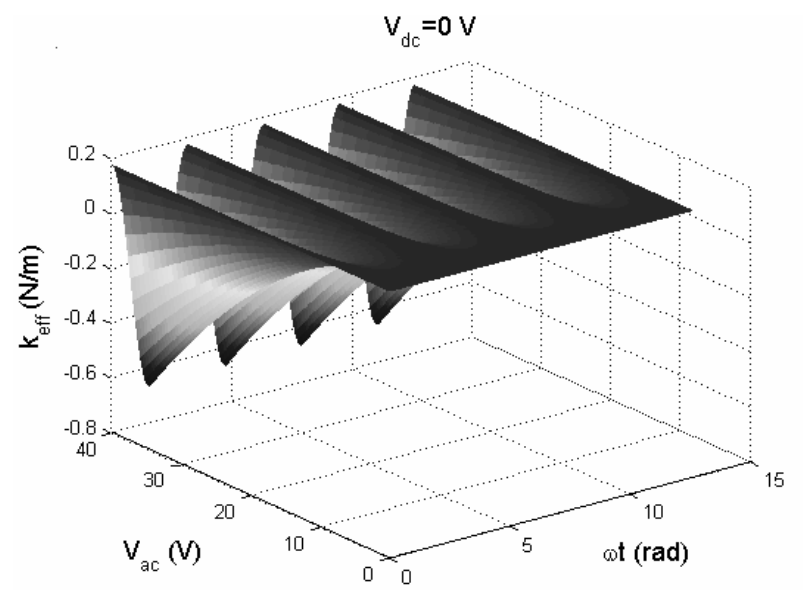

(a) $V_{d c}=0 \mathrm{~V}$ and $d_{0}=3 \mu m$

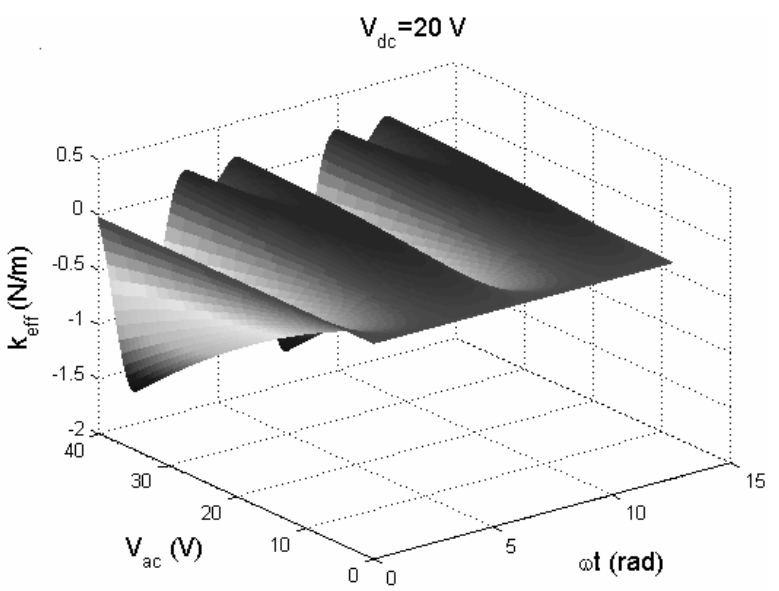

(b) $V_{d c}=20 \mathrm{~V}$ and $d_{0}=3 \mu m$

Fig. 2. Relationships between the effective stiffness and the dc bias voltage and the ac voltage: (a) $V_{d c}=0 \mathrm{~V}$ and $d_{0}=3 \mu m$; (b) $V_{d c}=20 \mathrm{~V}$ and $d_{0}=3 \mu \mathrm{m}$.

\subsection{Resonant frequency}

Resonant frequency is one of the important parameter confirming the exerted force in MEMS devices. It depends on both the electrostatic force and the deformation of the MEMS resonant structure. The nonlinearity in spring stiffness includes a combination of softening material nonlinearities and hardening geometric nonlinearities. The relative "spring-softening" or "spring-hardening" effect of the electrostatic force can be explained by the analytical expression (2). $F_{e}(t)$ is only a function of $V(t)$ at small displacements. However, with the increase of $V(t), x / d_{0}$ increases and, hence, $F_{e}(t)$ depends on $x$ as well. As a result, the electrostatic force $F_{e}(t)$ acts like a negative spring, which leads to "spring-softening".

It can be obtained from Eqs (1) and (2) that the effective stiffness $k_{e f f}=k-\frac{\varepsilon_{0} A_{0}}{d_{0}^{3}}\left(V_{d c}+V_{a c} \cos \omega t\right)^{2}$. The expression indicates that $k_{e f f}$ is a periodically time-varying function depending on the dc bias and ac voltages and gap. The electrostatic force softens the stiffness of the MEMS resonant structure periodically. Numerical simulations of the effective stiffness $k_{\text {eff }}$ can be seen from Fig. 2. Figure 2(a) illustrates that the effective stiffness decrease periodically with the increase of the frequency $\omega$ of the ac voltage and the variation increases with larger magnitude of the ac voltage $V_{a c}$ when $V_{d c}=0 \mathrm{~V}$ and $d_{0}=3 \mu \mathrm{m}$. When the dc bias voltage $V_{d c}$ is considered, $V_{d c}=20 \mathrm{~V}$, the effective stiffness also decrease periodically but with two-frequency components ( $\omega$ and $2 \omega)$ as shown in Fig. 2(b). It is demonstrated that the effective stiffness decreases periodically with the increase of the ac voltage and dc bias voltage.

Figure 3 gives the relationship between the first resonant frequency and dc bias voltage when the ac voltage $V_{a c}=0 \mathrm{~V}$ at different gaps for the MEMS resonator. It can be observed the "spring-softening" at three different gaps and indicated that the mechanical nonlinearity is absent due to the large deformation [28]. It is also shown that the resonant frequency increases with the increase of the gap at the same dc bias voltage. In addition, the resonant frequency is a monotonically decreasing function of increasing dc bias voltage when the ac voltage has not been considered in this case.

\subsection{Quality factor}

Squeeze film damping is one of the key parameters for the dynamic responses of the micro-devices. The larger the squeeze film damping is, the higher the noise level is led to. It can be obtained from Eq. (3) that the numerically simulations of the ratio of the fundamental resonant frequency in gas to that in vacuum $\omega_{\text {gas }} / \omega_{\text {vac }}$.

Figure 4 shows the ratio of resonant frequencies in vacuum and gas $\omega_{\text {gas }} / \omega_{v a c}$ as a function of the Reynolds number $R_{e}$ at different natural scaling parameter $\Pi$. It can be seen from Fig. 4 that $\omega_{\text {gas }} / \omega_{\text {vac }}$ is increasing with 


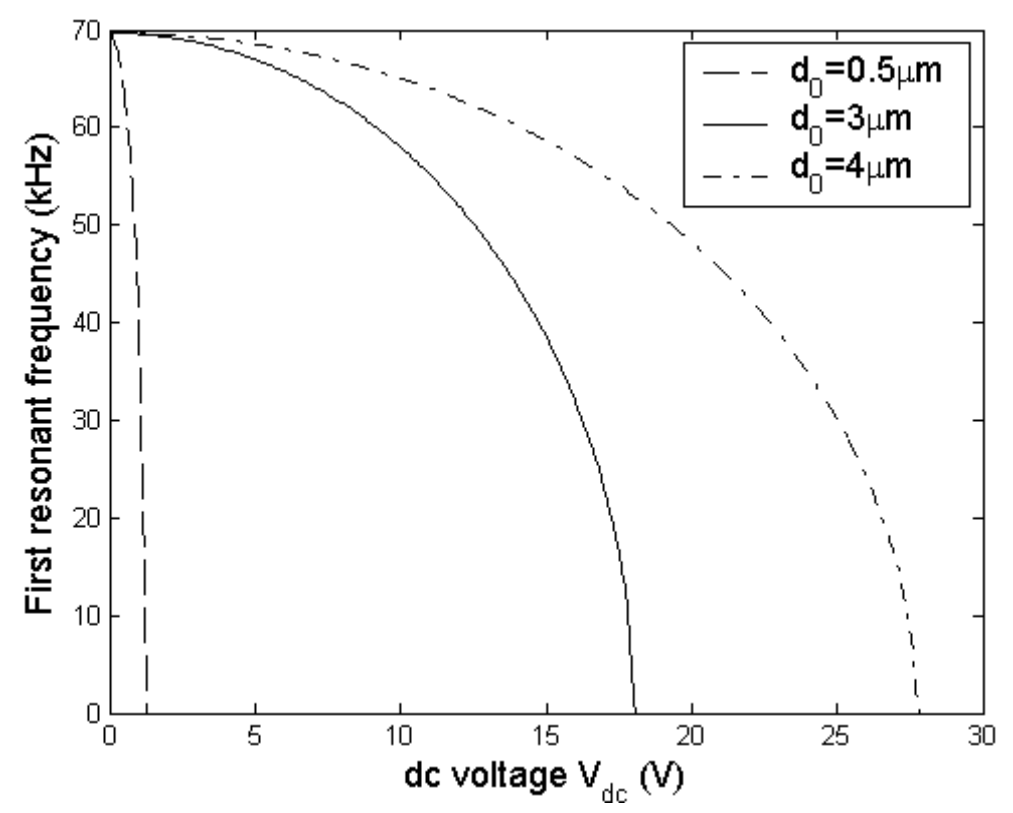

Fig. 3. Relationship between the first resonant frequency with the ac voltage at $V_{a c}=0 \mathrm{~V}$ and different gaps.

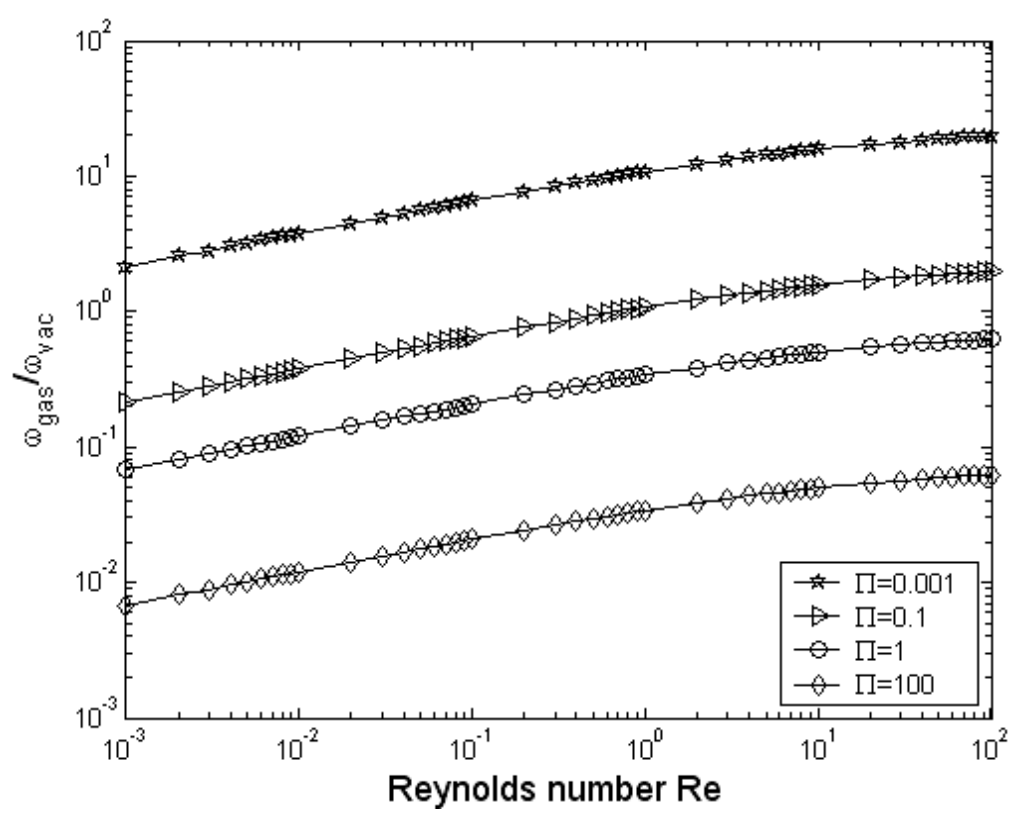

Fig. 4. Relationship between the vacuum resonant frequency $\omega_{v a c}$ and the resonant frequency in gas $\omega_{\text {gas }}\left(\omega_{\text {gas }} / \omega_{\text {vac }}\right)$ as a function of the Reynolds number $R_{e}$ at different natural scaling parameter $\Pi$.

the increase of Reynolds number $R_{e}$ and $\omega_{\text {gas }} / \omega_{\text {vac }}$ decreases with the increase of the scaling parameter $\Pi$. We can obtain from Eq. (4) that the quality factor $Q_{g a s}$ as a function of both natural scaling parameters, $R_{e}$ and $\Pi$, as illustrated in Fig. 5. It is indicated that the quality factor $Q_{\text {gas }}$ increases with the increase of the Reynolds number $R_{e}$ and the decrease of the natural scaling parameter $\Pi$. Meanwhile, when the natural scaling parameter $\Pi$ tends to be a larger value (in the case of $\Pi=10$ ), the quality factor $Q_{\text {gas }}$ has small change. As displayed in Fig. 5, when $\Pi$ changes from 10 to 100 , the quality factor $Q_{\text {gas }}$ has very small change. 


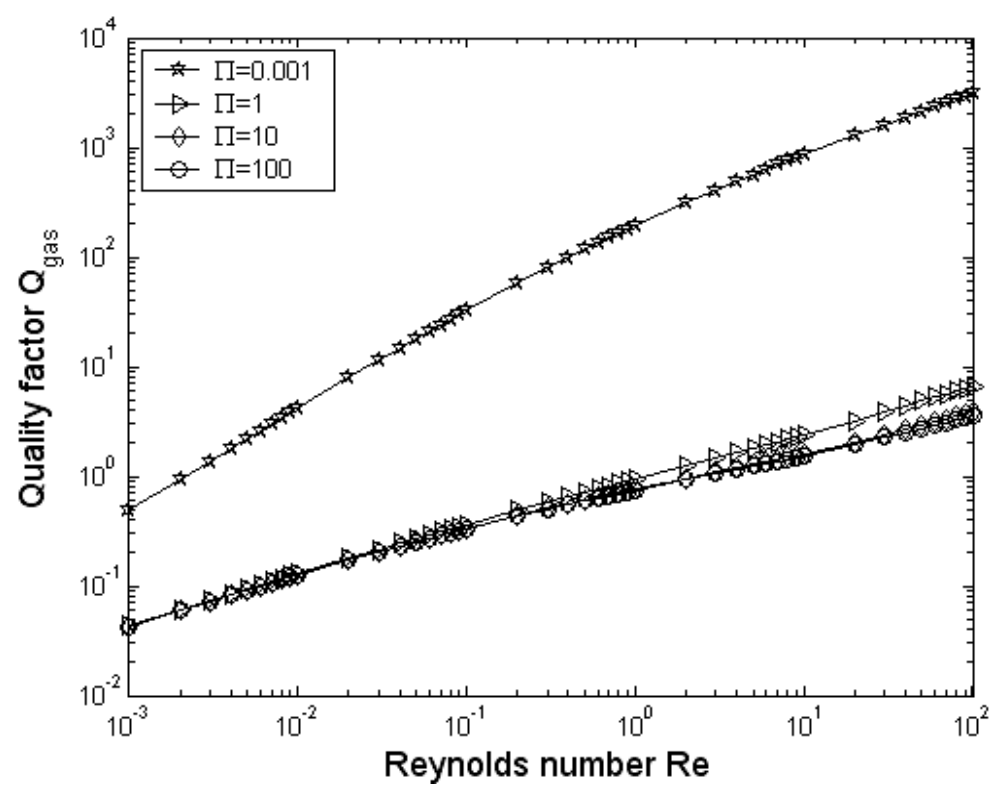

Fig. 5. Quality factor $Q_{g a s}$ as a function of the Reynolds number $R_{e}$ for the fundamental mode at different natural scaling parameter $\Pi$.

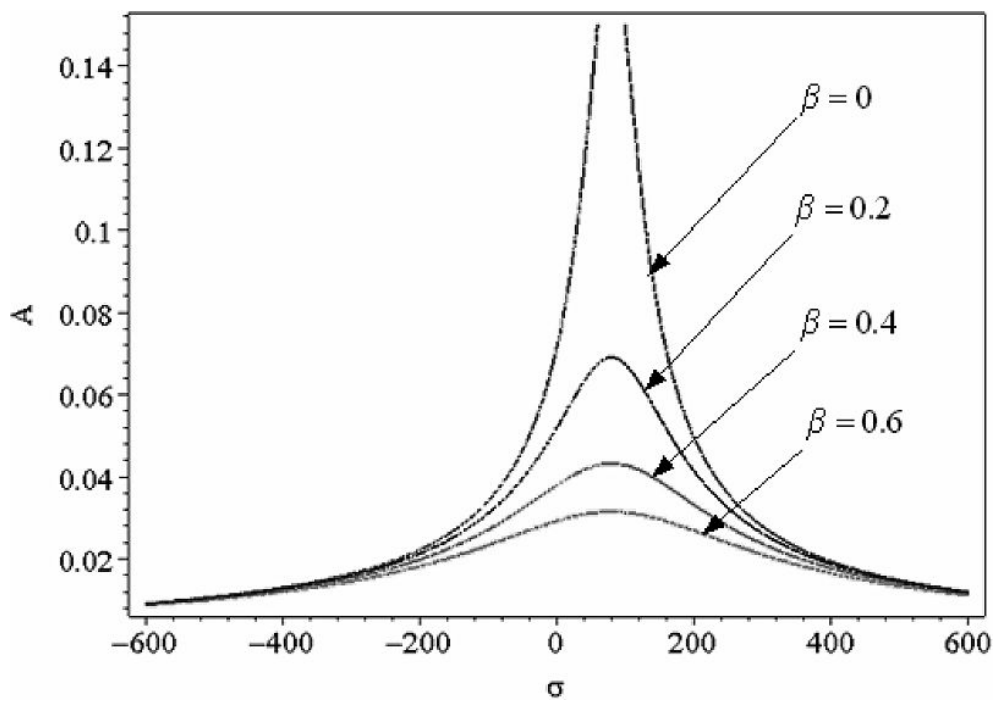

Fig. 6. Effect of squeeze film damping on frequency response at $\gamma=0.2, V_{d c}=30 \mathrm{~V}, V_{a c}=10 \mathrm{~V}$ and $\varepsilon=0.01$.

\subsection{Frequency responses}

It can be seen from Eq. (14) that the frequency response of a MEMS resonant structure is governed by the dc bias voltage, the ac voltage, as well as the squeeze film damping and the gap. To demonstrate the dependency of the steady-state behavior of the MEMS device, the frequency response for nonlinear squeeze film damping are illustrated graphically.

Figure 6 shows the variation of the squeeze film damping on the frequency response of the MEMS resonator. It should be noted that, in the absence of damping, the peak amplitude is infinite and the frequency response curve consists of two branches having as their asymptotes. With the increasing the damping, the amplitude of the oscillation can diminish as expected as possible. Therefore, decreasing the damping will result in the increase of the quality 


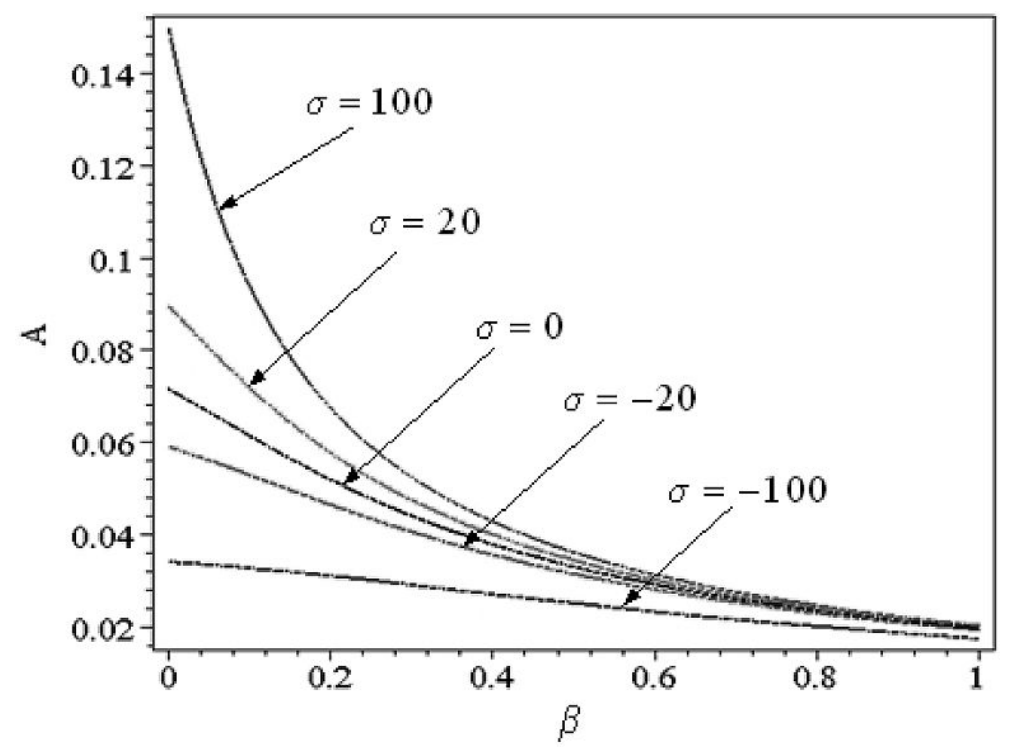

Fig. 7. Amplitude of the response as a function of the squeeze film damping for several values of $\sigma$.

factor of the resonant structure, which is considered a positive phenomenon. The effect of damping has two main roles in MEMS dynamics. It could be used to control the system remaining within the physical boundary $X<1$, and it must be as low as possible to increase the quality factor of the resonator.

Figure 7 illustrates the variation of the amplitude of the response with the squeeze film damping at several values of $\sigma$, in which the values of $\gamma=0.2, V_{d c}=30 \mathrm{~V}$ and $V_{a c}=10 \mathrm{~V}$ are the same for all curves. It can be found that these curves can be obtained directly from Eq. (14). It should be note that all the curves are single-valued depending on the value of $\sigma$. It shows from the frequency response that dc bias and ac voltages have softening effects and shift the resonant frequency to the lower values. In addition, the squeeze film damping has the least effect on resonant frequency with high effects on decreasing the peak amplitudes of the response of the resonator.

\subsection{Bifurcation and chaos behavior}

This section aims at numerically investigating nonlinear dynamics of a electrostatically actuated MEMS resonator.

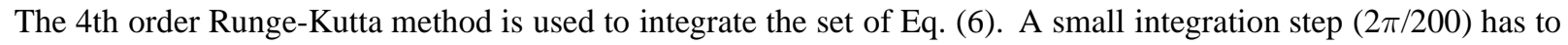
be chosen to ensure a stable solution and to avoid the numerical divergence at the points where derivatives of $F_{e}$ and $F_{s}$ are discontinuous. The effects of system parameters on the dynamic behavior of the cantilever vibrating system are investigated by using the bifurcation diagram, Poincaré maps, largest Lyapunov exponent, phase portraits, time histories and amplitude spectrum.

Squeeze film damping coefficient and ratio are the key parameters for the dynamic responses of the MEMS devices. The larger the squeeze film damping, the louder noise will exist. Considering the effect of squeeze film damping coupling with the electrostatic force on the system response and using the squeeze film damping as the control parameter which ranges from 0.0 to 1.0, Figs 8 and 9 display the bifurcation diagrams and largest Laypunov exponent maps of squeeze film damping ratio $\beta$ for the coupling nonlinear dynamic system with different applied voltages, i.e. $V_{d c}=0$ and $V_{a c}=40 \mathrm{~V}$, and $V_{d c}=40 \mathrm{~V}$ and $V_{a c}=40 \mathrm{~V}$.

It can be seen from Figs 8 and 9 that the system response changes between periodic and chaotic motion alternatively when $\beta<0.2$, and then it enters into synchronous with period- 1 when $\beta>0.2$. In addition, with the increase of dc bias voltage $V_{d c}$, we can find that the chaotic motion components increases and shifts to the lower squeeze film damping ratio. It is indicated that reasonable increase of dc bias voltage can reduce the effect of nonlinear squeeze film damping on the dynamic response of the coupling system.

Figures 10 and 11 show the Poincare maps and phase plane portraits of different squeeze film damping ratio $\beta$ on the responses of the coupling system with $V_{d c}=40 \mathrm{~V}$ and $V_{a c}=40 \mathrm{~V}$. The system response comes into synchronous 

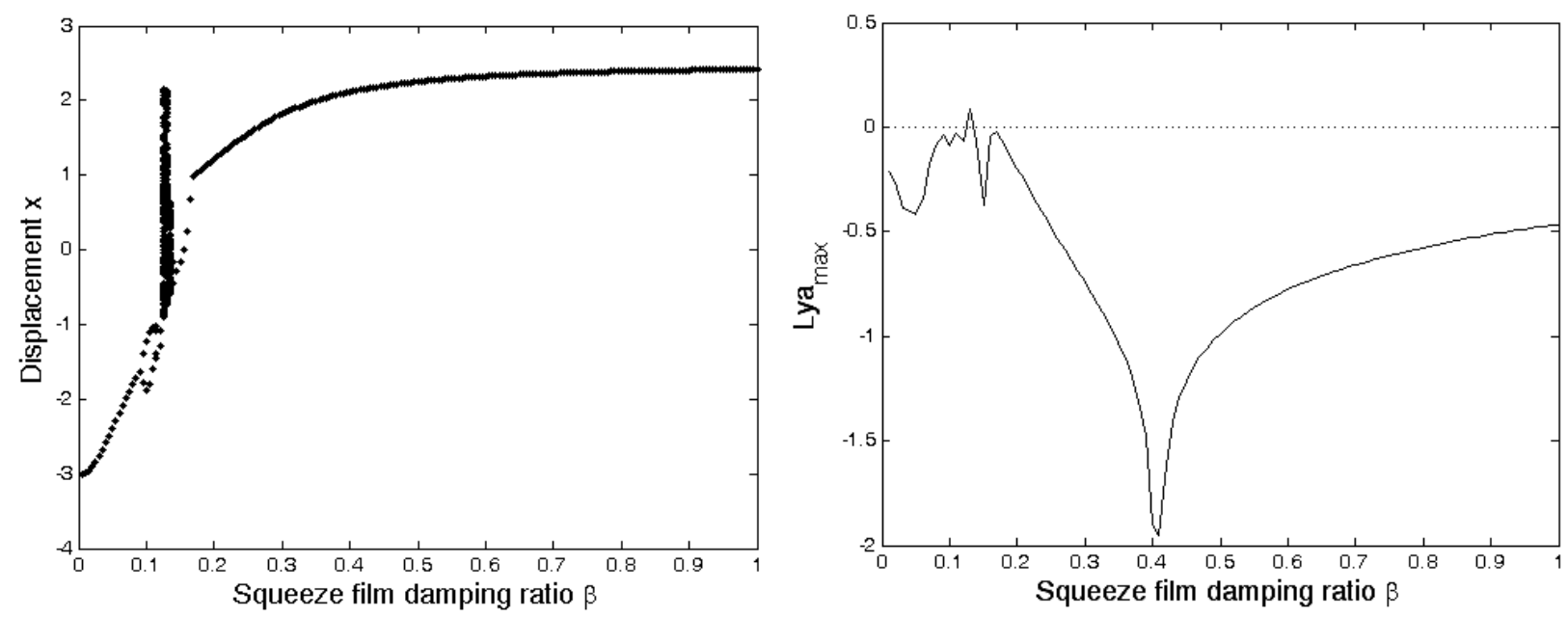

Fig. 8. Bifurcation diagram and largest Laypunov exponent map of $\beta$ for the coupling nonlinear dynamic system with $V_{d c}=0$ and $V_{a c}=40 \mathrm{~V}$.
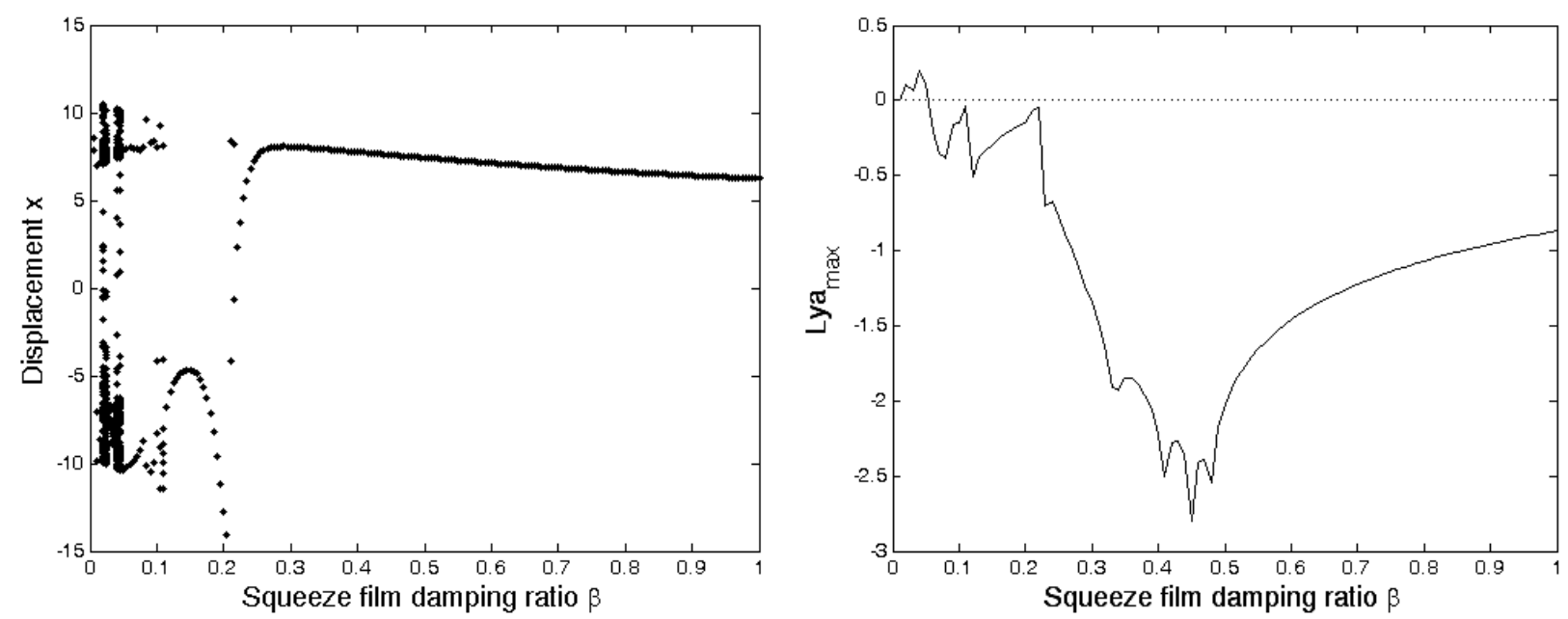

Fig. 9. Bifurcation diagram and largest Laypunov exponent map of $\beta$ for the coupling nonlinear dynamic system with $V_{d c}=40 \mathrm{~V}$ and $V_{a c}=40 \mathrm{~V}$.

motion with period-3 at $\beta=0.1$, as illustrated in Fig. 10(b), from chaotic motion at $\beta=0.04$, as displayed in Fig. 10(a), then it leaves synchronous motion with period-3 and enters synchronous motion with period-1 when $\beta>0.2$. At $\beta=0.04$, the chaos are shown in Fig. 10(a) and Fig. 11(a), the strange attractor has a fractal structure, the corresponding largest Lyapunov exponent is positive. With the increase of squeeze film damping ratio, we can find the period-3 motion with three isolated points in Poincare map and three circles in phase portrait at $\beta=0.1$, as illustrated in Fig. 10(b) and Fig. 11(b), the corresponding largest Lyapunov exponent becomes negative, which can be seen in Fig. 11. Therefore, the effect of squeeze film damping on the system response can not be neglected for MEMS structures at micro-scale.

The nonlinear dynamic characteristics of the electrostatically actuated MEMS resonators will alter with the change of the ac voltage and dc bias voltage, as shown in Fig. 12. It is indicated that the nonlinear dynamic system displays very rich nonlinear phenomena when the ac voltage and dc bias voltage have been investigated as the control parameters. With the increase of ac voltage and dc bias voltage, the system response changes from periodic motion to chaotic motion, and then returns to periodic motion. 


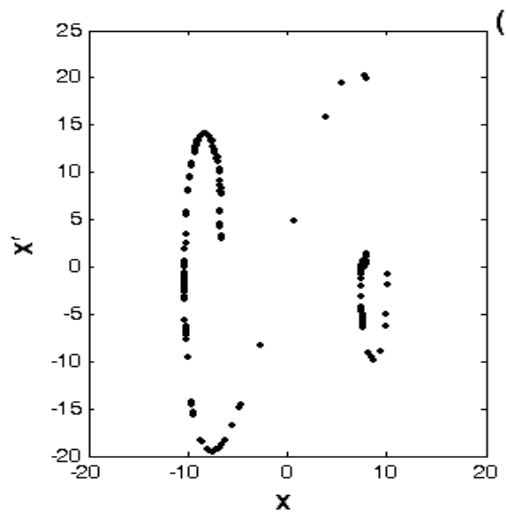

(a)

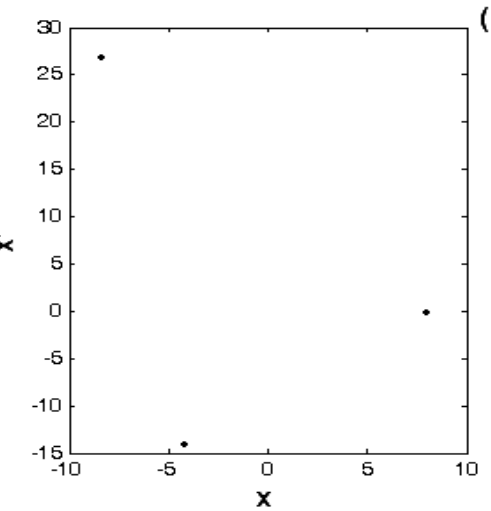

(b)

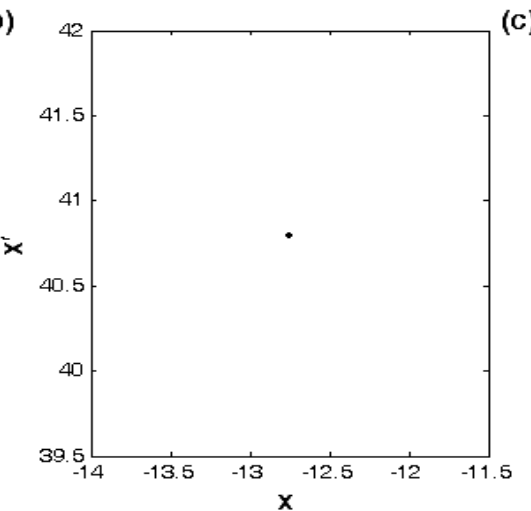

(c)

)

Fig. 10. Poincare maps on the responses of coupling system with different squeeze film damping ratio: (a) $\beta=0.04$; (b) $\beta=0.1 ;$ (c) $\beta=0.2$.

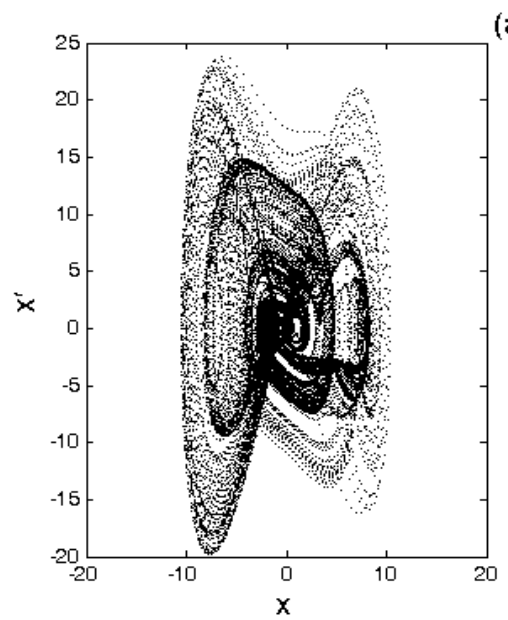

(a)

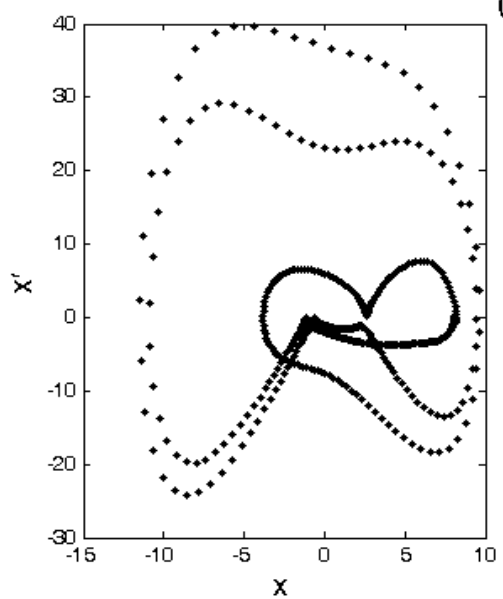

(b)

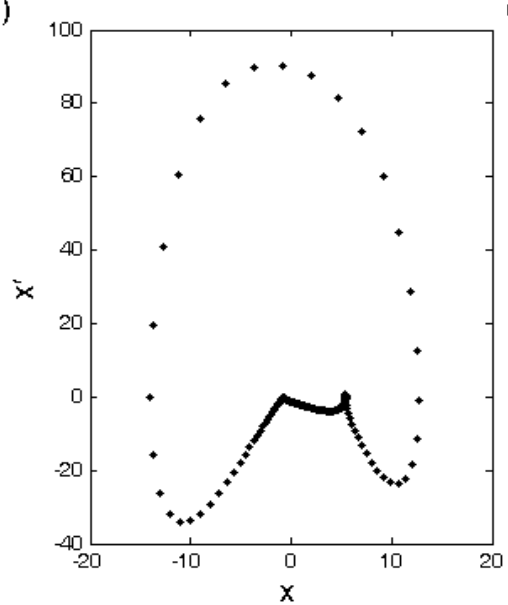

(c)

Fig. 11. Phase plane portraits on the responses of coupling system with different squeeze film damping ratio: (a) $\beta=0.04$; (b) $\beta=0.1$; (c) $\beta=0.2$.

\section{Conclusions}

We have presented analytical and numerical study of the effect of squeeze film damping on the dynamic responses and nonlinear dynamics of the electrostatically actuated MEMS cantilever resonators under combination resonant condition. The effect of nonlinear squeeze film damping on the frequency response, quality factor, resonant frequency and nonlinear dynamic characteristics of the dynamic system are provided using the bifurcation diagram, Poicare maps, largest Lyapunov exponent and phase portrait. The results show the effective stiffness decreases periodically with the increase of the ac voltage and dc bias voltage and the resonant frequency increases with the increase of the gap at the same dc bias voltage. In addition, the resonant frequency is a monotonically decreasing function of increasing dc bias voltage when the ac voltage has not been considered. It is demonstrated that decreasing the squeeze film damping will result in the increase of the quality of the MEMS resonators, which is regarded as a positive phenomenon. The dynamic system responses display very rich nonlinear dynamic characteristics under the effects of squeeze film damping, ac voltage and dc bias voltage, and show an alternate charging process beween the periodic motion and chaotic motion. The mass-spring-damping dynamic model of the electrostatically actuated MEMS resonator utilized in this work could be used to predict the dynamic responses for various parameters qualitatively and in some cases even quantitatively. Characteristic nonlinear dynamic steady-state behaviour is also very well predicted by this simplified dynamic model. It is indicated that both the analytical model and the numerical 

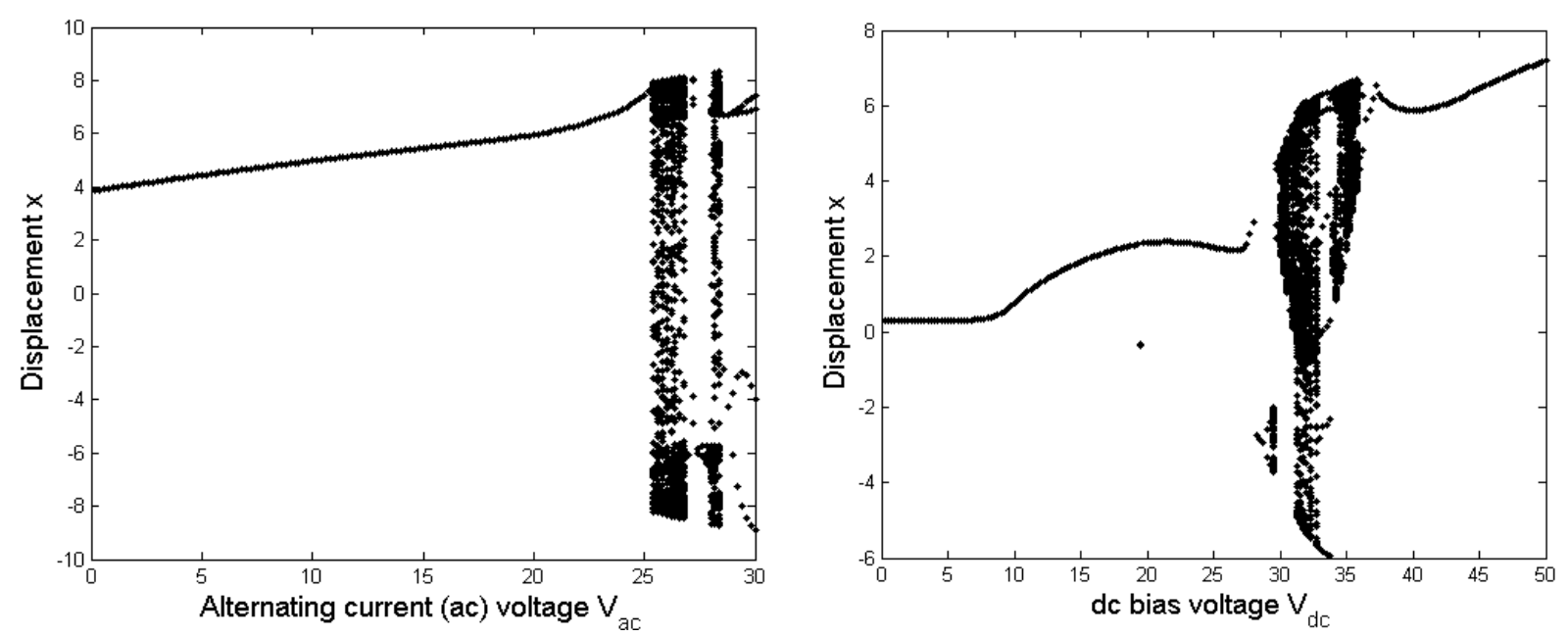

Fig. 12. Bifurcation diagram of (a) ac voltage $V_{a c}$ with $V_{d c}=40 \mathrm{~V}$, and (b) dc bias voltage $V_{d c}$ with $V_{a c}=20 \mathrm{~V}$ for the coupling nonlinear dynamic system.

simulations can be used very effectively to explain the effect of squeeze film damping on the dynamic responses of the MEMS resonators.

\section{Acknowledgements}

This work was supported by the National Science Foundation of China under Grant No. 10602033, the National Outstanding Youth Foundation of China under Grant No.10325209, and the Specialized Research Fund for State Key Laboratory of Mechanical System and Vibration under Grant No. MSV-MS-2008-15.

\section{References}

[1] M.S. El Naschie, Nanotechnology for the developing world, Chaos Solitons \& Fractals 30 (2006), 769-773.

[2] M.I. Younis, F. Alsaleem and D. Jordy, The response of clamped-clamped microbeams under mechanical shock, International Journal of Non-Linear Mechanics 42(4) (2007), 643-657.

[3] M.I. Younis, E.M. Abdel-Rahman and A.H. Nayfeh, Dynamic simulations of a novel RF MEMS switch, Proc 7th Int Conf on Modeling and Simulation of Microsystems: NanoTech (Boston, MA), 2004, pp. 287-290.

[4] L. Lin, C.T.C. Nguyen, R.T. Howe and A.P. Pisano, Microelectromechanical filters for signal processing, Proc IEEE Micro Electro Mechanical Systems MEMS '92 (Travemunde, Germany), 1992, pp. 226-231.

[5] W. Zhang, R. Baskaran and K.L. Turner, Effect of cubic nonlinearity on auto-parametrically amplified resonant MEMS mass sensor, Sensors and Actuators A: Physical 102 (2002), 139-150.

[6] D.W. Carr, S. Evoy, L. Sekaric, H.G. Craighead and J.M. Parpia, Parametric amplification in a torsional microresonator, Appl Phys Lett 77 (2000), 1545-1547.

[7] M. Zalalutdinov, A. Olkhovets, B. Ilic, D. Czaplewski and H.G. Craighead, Optically pumped parametric amplification for micromechanical oscillators, Appl Phys Lett 78 (2001), 3142-3144.

[8] M.F. Yu and G.J. Wagner, High order parametric resonance and nonlinear mechanics of nanowires, Technical Proc Nanotechnology Conf and Trade Show (San Francisco, CA) 3 (2003), 325-328.

[9] E.M. Abdel-Rahman and A.H. Nayfeh, Secondary resonances of electrically actuated resonant microsensors, J Micromech Microeng 13 (2003), 491-501.

[10] B.J. Gallacher, J.S. Burdess, A.J. Harris and J. Hedley, The application of parametric excitation to a micro-ring gyroscope, Technical Proc Nanotechnology Conf and Trade Show (Boston, MA) 1 (2004), 359-362.

[11] R. Lifshitz and M.C. Cross, Response of parametrically driven nonlinear coupled oscillators with application to micromechanical and nanomechanical resonator arrays, Phys Rev B 67 (2003), 1343021.

[12] B.J. Gallacher, J.S. Burdess and K.M. Harish, A control scheme for a MEMS electrostatic resonant gyroscope excited using combined parametric excitation and harmonic forcing, J Micromech Microeng 16 (2006), 320-331.

[13] M. Napoli, R. Baskaran, K.L. Turner and B. Bamieh, Understanding mechanical domain parametric resonance in microcantilevers, Proc IEEE 16th Int Annual Conf on MEMS, 2003, pp. 169-172. 
[14] E. Buks and M.L. Roukes, Electrically tunable collective response in a coupled micromechanical array, J Microelectromech Syst 11 (2002), 802-807.

[15] Y.C. Hu, C.M. Chang and S.C. Huang, Some design considerations on the electrostatically actuated microstructures, Sensors and Actuators A: Physical 112 (2004), 155-161.

[16] R. Baskaran and K.L. Turner, Mechanical domain coupled mode parametric resonance and amplification in a torsional mode micro electro mechanical oscillator, J Micromech Microeng 13 (2003), 701-707.

[17] K.L. Turner, S.A. Miller, P.G. Hartwell, N.C. MacDonald, S.H. Strogatz and S.G. Adams, Five parametric resonances in a micromechanical system, Nature 396 (1998), 149-152.

[18] S. Krylov, I. Harari and Y. Cohen, Stabilization of electrostatically actuated microstructures using parametric excitation, J Micromech Microeng 15 (2005), 1188-1204.

[19] M.I. Younis and A.H. Nayfeh, A study of the nonlinear response of a resonant microbeam to electric actuation, Nonlinear Dynamics 31 (2003), 91-117.

[20] W.M. Zhang and G. Meng, Nonlinear dynamical system of micro-cantilever under combined parametric and forcing excitations in MEMS, Sensors and Actuators A: Physical 119 (2005), 291-299.

[21] A.H. Nayfeh and M.I. Younis, Dynamics of MEMS resonators under superharmonic and subharmonic excitations, J Micromech Microeng 15 (2005), 1840-1847.

[22] W.M. Zhang, G. Meng and D. Chen, Stability, nonlinearity and reliability of electrostatically actuated MEMS devices, Sensors 7 (2007), 760-796.

[23] R.M.C. Mestrom, R.H.B. Fey, J.T.M. Van Beek, K.L. Phan and H. Nijmeijer, Modelling the dynamics of a MEMS resonator: simulations and experiments, Sensors and Actuators A: Physical 142 (2008), 306-315.

[24] N. Kacem, S. Hentz, D. Pinto B. Reig and V. Nguyen, Nonlinear dynamics of nanomechanical beam resonators: improving the performance of NEMS-based sensors, Nanotechnology 20 (2009), 275501(11pp).

[25] F.M. Alsaleem, M.I. Younis and H.M. Ouakad, On the nonlinear resonances and dynamic pull-in electrostatically actuated resonators, $J$ Micromech Microeng 19 (2009), 045013(14pp).

[26] W.M.Zhang and G. Meng, Nonlinear dynamic analysis of electrostatically actuated resonant MEMS sensors under parametric excitation, IEEE Sensors Journal 7(3) (2007), 370-380.

[27] S.K. De and N.R. Aluru, Complex nonlinear oscillations in electrostatically actuated microstructures, Journal of Microelectromechanical Systems 15(2) (2006), 355-369.

[28] S.K. De and N.R. Aluru, Full-Lagrangian schemes for dynamic analysis of electrostatic MEMS, Journal of Microelectromechanical Systems 13(5) (2004), 737-758.

[29] S. Liu, A. Davidson and Q. Lin, Simulating nonlinear dynamics and chaos in a MEMS cantilever using Poincare mapping, IEEE the 12th International Conference on Solid State Sensors, Actuators and Microsystems, Boston, June 8-12, 2003, pp. 1092-1095.

[30] J.E. Sader, Frequency response of cantilever beams immersed in viscous fluids with applications to the atomic force microscope, $J$ Appl Phys 84 (1998), 64-76.

[31] S. Basak and A. Raman, Hydrodynamic loading of microcantilevers vibrating in viscous fluids, J Appl Phys 99 (2006), 114906.

[32] J.B. Starr, Squeeze-film damping in solid-state accelerometers, Proc. IEEE Solid State Sensor and Actuator Workshop, Hilton Head Island, SC, USA, June 4-7, 1990, pp. 44-47.

[33] P.B. Chu, P.R. Nelson, M.L. Tachiki and K.S. Pister, Dynamics of polysilicon parallel-plate electrostatic actuator, Sensors and Actuators A: Physical 52 (1996), 216-220.

[34] A.H. Nayfeh, Perturbation methods, Wiley, New York, 1973. 

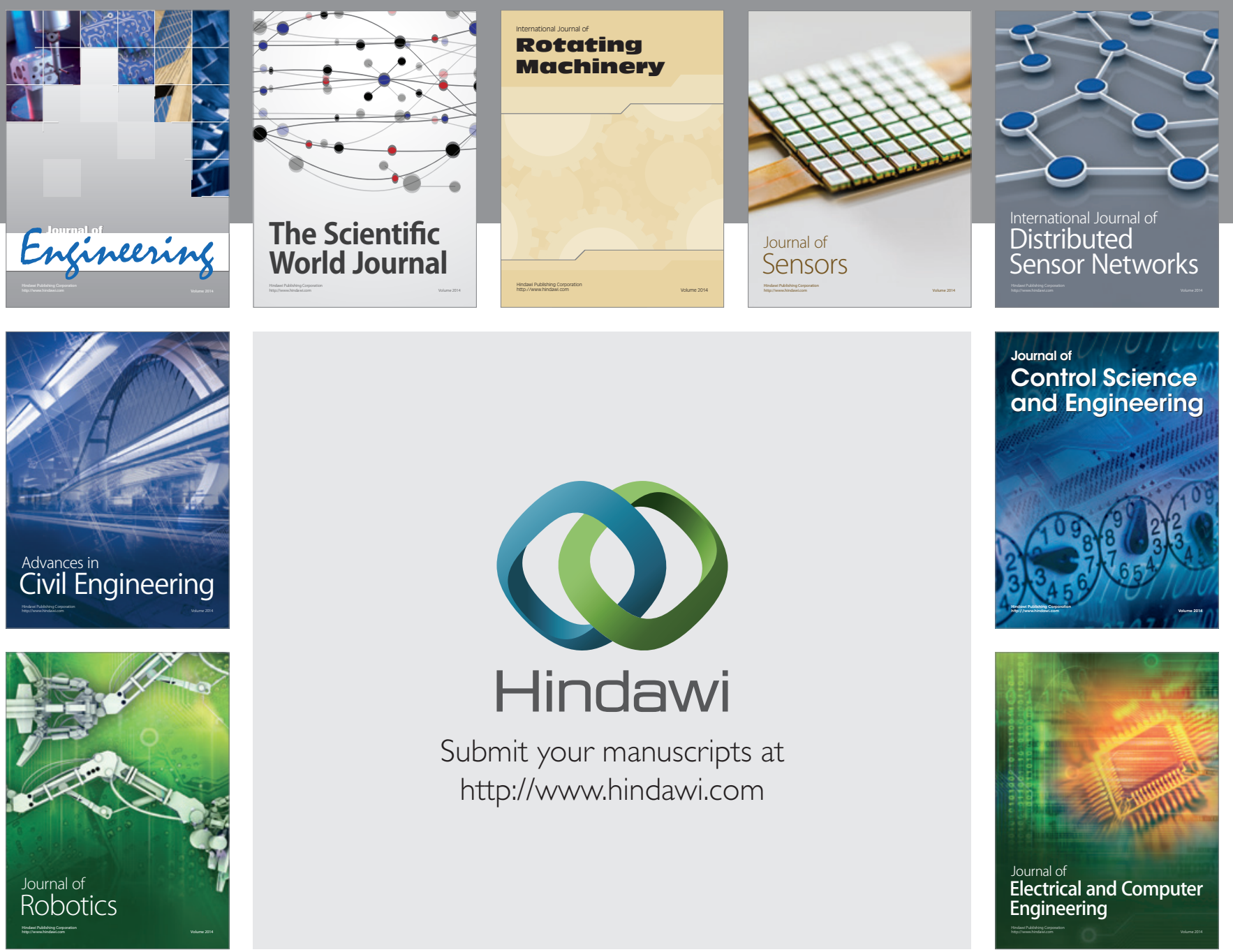

Submit your manuscripts at

http://www.hindawi.com
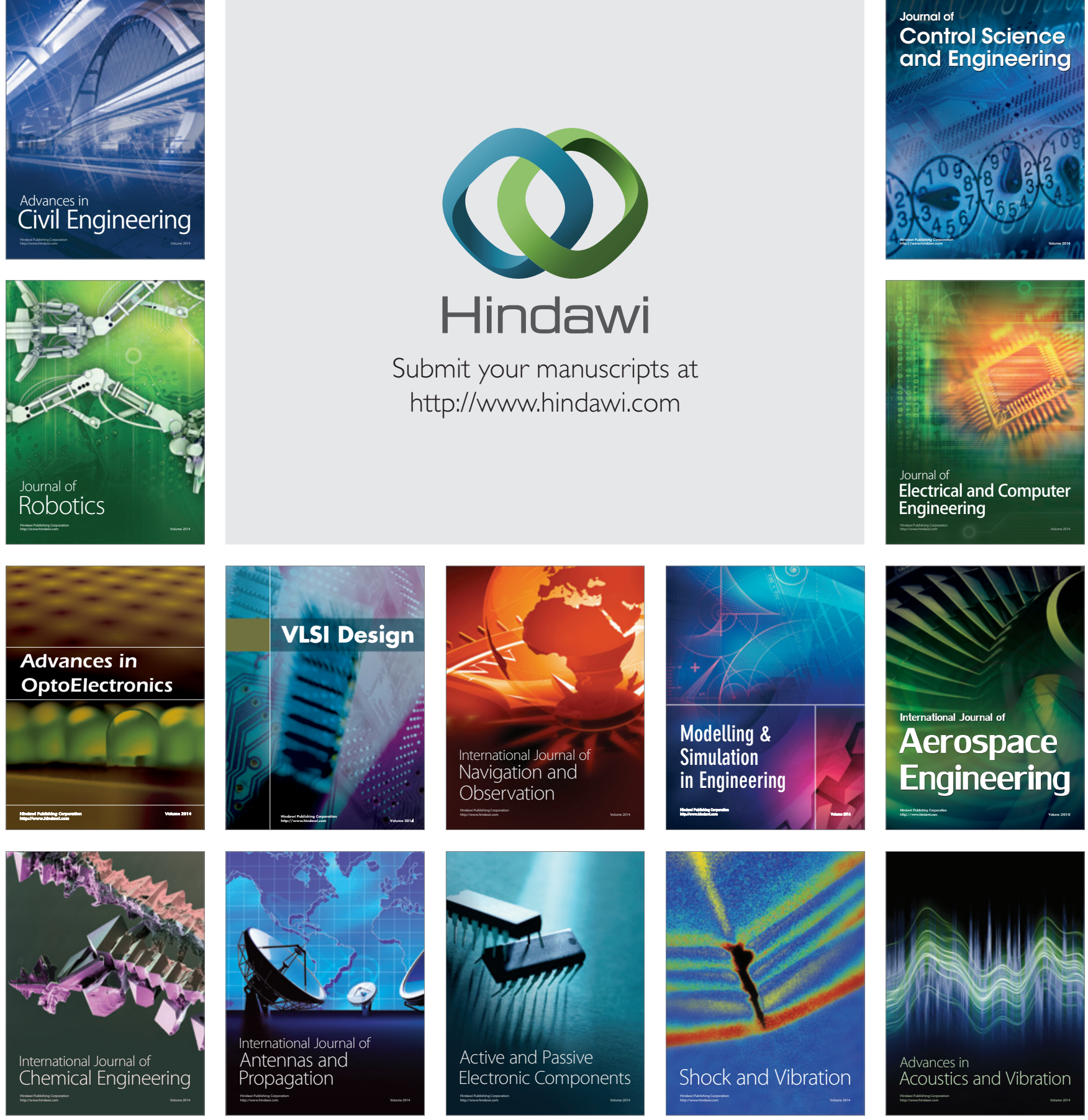